\title{
Differentiation profile of brain tumor stem cells: a comparative study with neural stem cells
}

\author{
Quan Bin Zhang ${ }^{1}$, Xiao Yan Ji ${ }^{1}$, Qiang Huang ${ }^{1}$, Jun Dong ${ }^{1}$, Yu De Zhu ${ }^{1}$, Qing Lan ${ }^{1}$ \\ ${ }^{1}$ Neurosurgical Department and Brain Tumor Research Laboratory, Second Affiliated Hospital of Suzhou University, Suzhou 215004, \\ China
}

Understanding of the differentiation profile of brain tumor stem cells (BTSCs), the key ones among tumor cell population, through comparison with neural stem cells (NSCs) would lend insight into the origin of glioma and ultimately yield new approaches to fight this intractable disease. Here, we cultured and purified BTSCs from surgical glioma specimens and NSCs from human fetal brain tissue, and further analyzed their cellular biological behaviors, especially their differentiation property. As expected, NSCs differentiated into mature neural phenotypes. In the same differentiation condition, however, BTSCs exhibited distinguished differences. Morphologically, cells grew flattened and attached for the first week, but gradually aggregated and reformed floating tumor sphere thereafter. During the corresponding period, the expression rate of undifferentiated cell marker CD133 and nestin in BTSCs kept decreasing, but 1 week later, they regained ascending tendency. Interestingly, the differentiated cell markers GFAP and $\beta$-tubulinIII showed an expression change inverse to that of undifferentiated cell markers. Taken together, BTSCs were revealed to possess a capacity to resist differentiation, which actually represents the malignant behaviors of glioma.

Cell Research (2006) 16:909-915. doi: 10.1038/sj.cr.7310104; published online 7 November 2006

Keywords: brain tumor stem cell, neural stem cell, differentiation

\section{Introduction}

Glioma is the most common neoplasm of central nervous system in human and remains difficult to cure despite rapid advances in diagnostic imaging techniques and multimodality therapy. For the past decades, huge endeavors have been dedicated to elucidate the cellular and molecular mechanisms of this intractable disease; however, no substantial breakthrough has been achieved due to the fact that brain tumor mass is composed of heterogeneous combination of cells. According to Reya et al. [1], among tumor cell population, there always exist stem cells that are rare but critical in tumorigenesis. Following the identification of tumor stem cells from leukemia and breast cancer $[2,3]$, brain tumor stem cells (BTSCs), as recently

Correspondence: Qiang Huang

Tel: +86-512-67783937;

E-mail: szqhdnah@pub.sz.jsinfo.net

Received 18 May 2006; revised 23 August 2006; accepted 19 September 2006; published online 7 November 2006 reported, were cultured from both surgical specimens and cultured glioma cell lines, according to the following three lines of evidence: (1) expressing known neural stem cell (NSC) surface marker CD133 and nestin, and generating spheres that are morphologically indistinguishable from neurospheres; (2) self-renewing and proliferating; and (3) producing differentiated progeny in vitro or recapitulating the parental tumor mass when implanted into the immunodeficient animals [4-9]. Singh et al. [4] observed that, under differentiation condition, all tumor spheres grew attached as monolayer, lacking expression of undifferentiated cell marker CD133 and nestin. Tumor stem cells have the characteristic of forming tumor sphere; however, the fact that only a small proportion of cells within tumor spheres are true stem cells makes it necessary to isolate and focus on tumor stem cells if the exact biological behaviors of this special cell fraction are to be fully understood. In this study, we purified BTSCs from brain tumor spheres, and isolated human NSCs, the mostly likely glioma cell-of-origin [1012], from neurospheres, and made a comparative study to specify the differentiation profile of BTSCs. 


\section{Material and Methods}

\section{Tumor samples and human fetal brain tissue}

Tumor tissues were obtained from a 52-year-old female patient undergoing twice operations within 2 months for rapid relapse. Both primary and recurrent lesions were pathologically diagnosed as mixed glioma consisting of anaplastic ependymoma and astrocytoma. Human fetal brain tissue was obtained from the consent-informed patient receiving induced abortion in accordance with protocols approved by the Research Ethics Board at the Second Affiliated Hospital of Suzhou University.

\section{Tumor sphere and neurosphere culture}

Briefly, the fresh tumor specimens were washed, deprived of vessels, acutely dissociated in PBS and subjected to enzymatic dissociation as described previously [2]. Cells were suspended in defined stem cell medium consisting of Dulbecco's modified Eagle's medium/F-12 (Gibco), $\mathrm{N}_{2}$ supplement (Gibco), EGF (20 ng/ml, Invitrogen) and bFGF (20 ng/ml, Gibco), and plated at a density of $5 \times 10^{6}$ live cells per $75 \mathrm{~cm}^{2}$ flask. Cells were fed by changing half of the medium every 3 days. Brain tissue from a 1-month-old embryo was subjected to the same procedure as that mentioned above to get neurospheres. Both tumor spheres and neurospheres were enzymatically dissociated into single cells for expanded culture when they were large in size and high in density.

\section{Purification of BTSCs and NSCs}

Neurospheres, tumor spheres as well as cells differentiated from tumor spheres were harvested, subjected to enzymatic dissociation and resuspended in $1 \times$ PBS containing BSA and $2 \mathrm{mM}$ EDTA. Magnetic labeling with $1 \mathrm{ml}$ CD133/1 Microbead/1 million cells was performed using the CD133 Cell isolation kit (Miltenyi Biotec). Cell sorting was carried out on the auto-MACS machine (Miltenyi Biotec), with collected positive and negative fractions. The efficiency of cell sorting was further evaluated by flow cytometry (BD FACS Vantage).

\section{Subsphere forming assay}

Firstly, both tumor spheres and neurospheres were dissociated into single cells and transferred to 96-well plates at density ranging from 10 cells to 200 cells per well. Cultures were fed by changing $50 \mathrm{ml}$ of defined stem cell medium every 3 days till day 10 . Secondly, both tumor spheres and neurospheres were subject to differentiation con- dition (in medium containing 10\% FBS) for 7 days, then transferred to and cultured in defined stem cell medium. Thirdly, sorted CD $133^{+}$ tumor cells and CD133- tumor cells were plated to 96-well plates containing defined stem cell medium. Formation of subspheres was examined with phase-contrast microscopy.

\section{Differentiation assay of BTSCs and NSCs}

Immunofluorescent staining of BTSCs and NSCs Purified BTSCs and NSCs were plated onto glass coverslips coated in poly-L-ornithine (Sigma) in a 24-well plate. Differentiation was induced with culture medium added with $10 \%$ FBS. Coverslips were taken out on days 0 (4 h later), 3, 7 and 10, fixed with $4 \%$ paraformaldehyde and immunostained with antibodies against CD133 (Miltenyi Biotec), nestin (BDbioscience Co.), GFAP (Santa Cruz Co.) and MAP2. Laser scanning confocal microscope (Carl Zess Co.) was employed to examine the costaining of the above cell markers during differentiation of BTSCs and NSCs.

Detection of the expressing rates of cell surface markers BTSCs and NSCs were transferred to the differentiation condition mentioned above, and morphological changes of cells were observed under phase-contrast microscopy. On days $0,3,7,10$ and 21 , cells were collected and checked with flow cytometry to determine the expressing rates of cell surface markers, including CD133, nestin, GFAP and $\beta$-tubulinIII (BDbioscience Co.). Each test was repeated three times, and the results were processed with SSPS software.

\section{Results}

Tumor spheres and subspheres were successfully cultured and confirmed to be exclusively generated by $C D 133^{+}$ glioma cell

Within 24-48 h of culturing of single-cell suspensions made from both primary and recurrent tumor tissues, neurosphere-like clusters, usually termed tumor spheres, emerged and increased in diameter and density steadily (Figure 1A-1C). Neurospheres were also observed after the same duration, but grew slowly when compared with tumor spheres (data not shown). After tumor spheres were harvested and subjected to subsphere forming assay by lim-
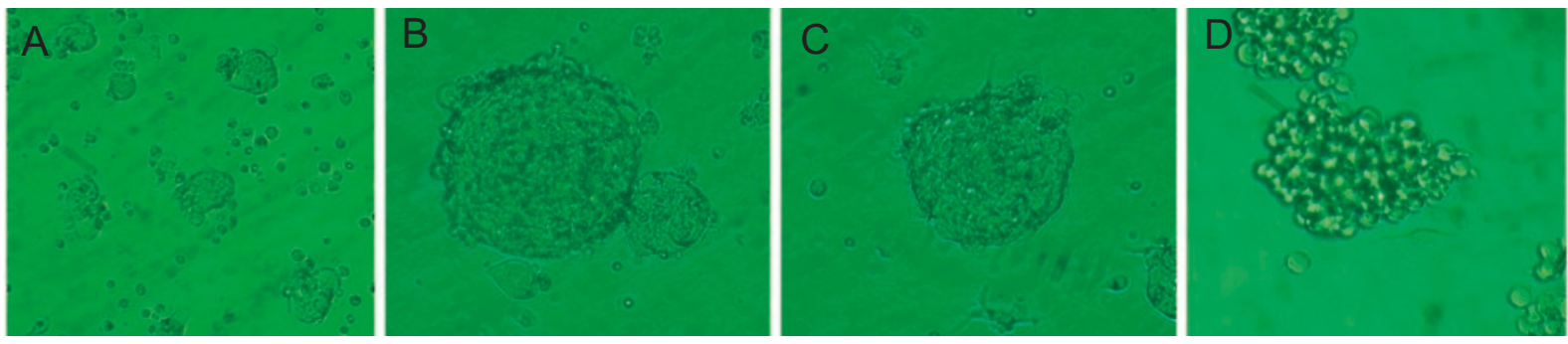

Figure 1 Both primary and recurrent gliomas from the same patient formed neurosphere-like colonies, termed tumor spheres. (A) Small tumor spheres emerged within $48 \mathrm{~h}$ of culturing of single-cell suspension (magnification $40 \times$ ). (B and C) Tumor spheres are shown from primary and recurrent gliomas, respectively (magnification $10 \times$ ). (D) Tumor spheres maintained for long term in vitro seem to morphologically vary from primary tumor spheres (magnification $10 \times$ ). 

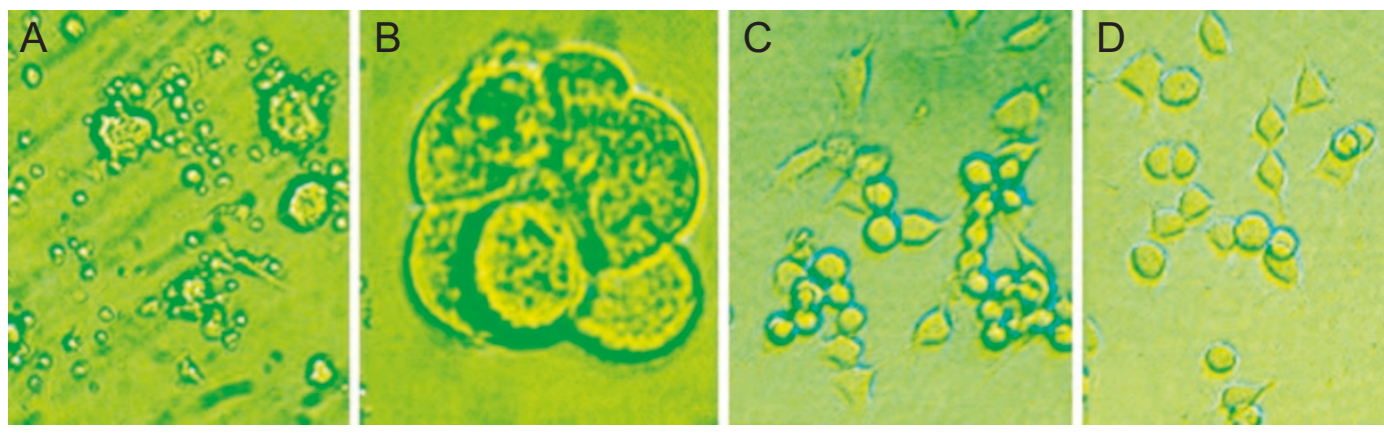

Figure 2 Comparison of clonogenicity between CD133 ${ }^{+}$and CD133- glioma cells. (A) Single CD133 ${ }^{+}$cells purified with magnetic cell sorting system produced small tumor spheres within $48 \mathrm{~h}$ of culturing in defined stem cell medium (magnification $10 \times$ ). (B) One tumor sphere is shown formed by one $\mathrm{CD}_{133^{+}}$glioma cell (magnification $40 \times$ ). (C and D) CD133- glioma cell could not generate tumor sphere but grew flattened and attached in the same culture condition (magnification 10×).

ited dilution, subspheres appeared as expected. After being in differentiation condition for 7 days, both tumor sphere and neurosphere cells were observed to migrate from spheres and become flat and adherent. However, when they were re-switched to defined stem cell medium, a difference occurred: monolayer adherent cells from tumor spheres gradually retracted their processes, floated up and formed rolling spheres again, whereas cells from neurospheres kept their morphology unchanged. This interesting phenomenon was also described by Yuan et al. [13]. Since CD133 is regarded as the putative surface marker for BTSCs $[4,9,13]$, single $\mathrm{CD}_{133^{+}}$glioma cell should be able to form tumor sphere. To prove this, we plated $\mathrm{CD} 133^{+}$cells and $\mathrm{CD} 133^{-}$glioma cells into 96 wells containing defined stem cell medium. As a result, $\mathrm{CD} 133^{+}$glioma cells proliferated in culture as floating spheres, whereas CD133- glioma cells got attached to the culture dishes, and unlike $\mathrm{CD} 133^{+}$glioma cells, they did not form spheres (Figure 2).

\section{BTSCs were kept for long term}

At the closure of this experiment, tumor spheres from both primary and recurrent tumor tissues have been kept for more than 18 months and expanded for more than 150 passages. Impressively, about 4 months after the primary sphere culture, not only did the morphology of the tumor spheres alter but also the proliferating rate of tumor sphere cells was obviously increased, with the doubling time shortened from 1 week to 3 or 4 days (Figure 1D and data not shown). Nevertheless, NSCs could only be maintained for no more than 3 months and no growth acceleration was observed.

BTSCs and NSCs were purified effectively with magnetic bead cell sorting system

CD133, a putative cell surface marker of human NSCs, was recently found to identify an exclusive subpopulation of glioma cells that possess stem-like activity [4]. We made an attempt to isolate BTSCs by magnetic cell sorting with antibody against $\mathrm{CD} 133$. As a result, $\mathrm{CD} 133^{+}$cells sorted from tumor spheres accounted for a minor fraction of tumor cell population, ranging from $3.8 \%$ to $4.0 \%$; however, when tumor spheres differentiated, the frequency of $\mathrm{CD} 133^{+}$cells decreased sharply to $1.8-2.0 \%$. The efficiency of magnetic
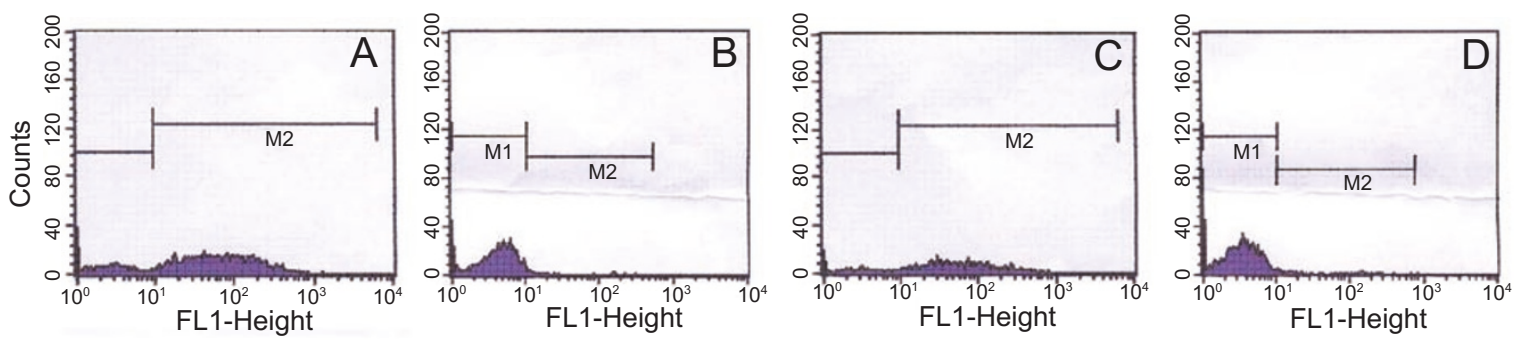

Figure 3 The efficiency of magnetic bead cell sorting was evaluated by flow cytometry, with the first peak (gate M1) representing cells negative for CD133- phycoerythrin expression, and the second peak (gate M2) representing CD133 ${ }^{+}$cells. (A and B) Purity was $83.02 \%$ in $\mathrm{CD}_{133^{+}}$glioma cell fraction and $97.7 \%$ in CD133- glioma cell fraction. (C and D) Purity of sorted CD133 ${ }^{+}$and CD133neural cells reached $85.06 \%$ and $96.68 \%$, respectively. 


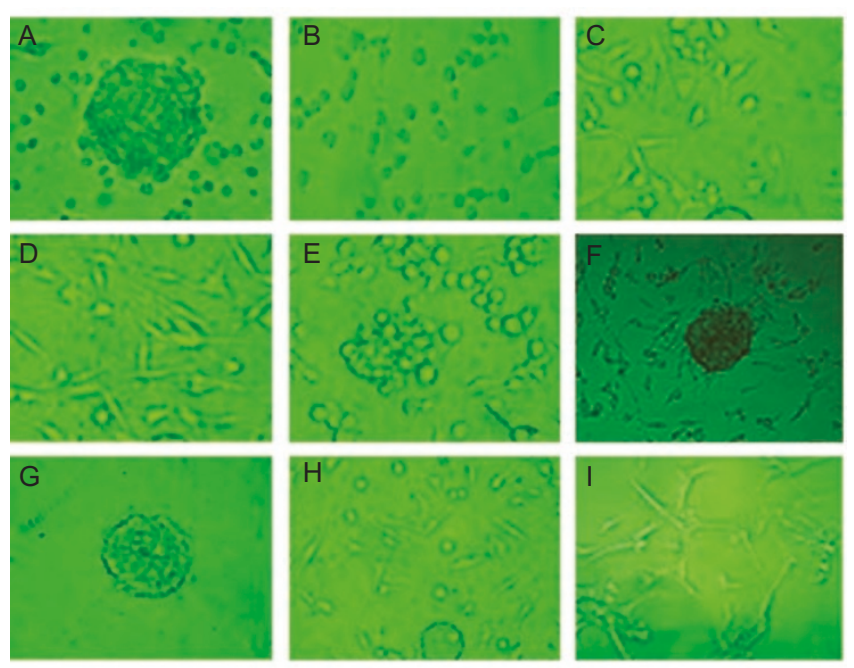

Figure 4 In differentiation condition, BTSCs and NSCs exhibited apparent difference in morphological changes (magnification $10 \times$ ). (A-F) Reforming of tumor spheres by BTSCs in differentiation condition: tumor spheres in defined stem cell medium (A); sorted single BTSC suspension was transferred to differentiation condition (B); cells became fusiform and adherent on day 4 of differentiation (C); cells were mostly fusiform on day 7 (D); cells began to retract processes and float up by day $10(\mathbf{E})$; and tumor sphere was reformed 21 days later (F). (G-I) Morphological changes of NSCs in the same differentiation condition: neurosphere in defined stem cell medium (G); on day 7 of differentiation, NSCs varied in morphology, but were predominantly fusiform (H); and neurosphere did not recur like tumor sphere by day 21 (I).

cell sorting was evaluated by flow cytometry, showing that the purity exceeded $80 \%$ in $\mathrm{CD} 133^{+}$cell fraction and $95 \%$ in CD133 cell fraction. Isolation of NSCs from neurospheres with magnetic cell sorting was also performed, with satisfactory outcomes (detailed in Figure 3).

\section{BTSCs demonstrated intrinsic potential to resist differen- tiation}

For the first week in differentiation condition, both NSCs and BTSCs gradually adhered to the bottom of flasks and morphologically became flat and fusiform. With maintenance of differentiation condition, NSCs still grew attached, but BTSCs gradually retracted their processes, floated up and reformed suspending spheres (Figure 4). At the beginning of differentiation, immunofluorescence detected by laser scanning confocal microscope demonstrated costaining of undifferentiated cell surface markers (CD133 and nestin), showing lack of differentiated cell markers (GFAP and MAP2) on BTSCs. However, on day 10 of differentiation, BTSCs still exhibited positive costaining of CD133 and nestin, although apparent staining of GFAP emerged (Figure 5). In NSCs, the staining of CD133 and nestin gradually faded with differentiation, and almost disappeared by day 10. The differentiation assay with flow cytometry confirmed the immunofluorescent results. In the first week of differentiation, the expression rates (number of marker positive cells/total number of examined cells) of undifferentiated cell markers CD133 and nestin in BTSCs kept decreasing as expected, but increased from day 10 (Figure 6A). During the corresponding period, differentiated markers GFAP and $\beta$-tubulin experienced an "up-down" tendency, which was inverse to that of undifferentiated markers (Figure 6A). The same experiment was also performed in NSCs; however, in this case, expression rates of CD133 and nestin kept decreasing, while those of GFAP and $\beta$-tubulin kept increasing, and no turning point emerged (Table 1).

\section{Discussion}

It is traditionally believed that glioma may develop from NSCs that undergo abnormal differentiation or from differentiated cell types that acquire malignance by de-differentiating in response to oncogenic mutation [14-17]. Most recently, the study on tumorigenesis of glioma was further complicated by cell-cell fusion theory, in which mature cells could gain de-differentiation through fusion with stem cells [18-20]. Although the target cell of transformation mutation is still under investigation, there is considerable evidence that glioma is most likely derived from NSCs: (1) fewer mutations may be required to maintain self-renewal than to activate self-renewal ectopically, that is, NSCs is more sensitive to oncogenic stimuli; (2) NSCs often persist for relatively long term, which means there is a much greater opportunity for mutation to accumulate in NSCs [10-11, 21-22]. The successful identification of BTSCs from solid glioma with the technique used to identify NSCs supports the linkage between glioma and NSCs, as both of them (1) express CD133; (2) form rolling spheres; (3) possess the capacity for self-renewal, proliferation and multi-differentiation; and (4) share similar signaling pathway in cell cycle progression [23-26]. The similarity shared by BTSCs and NSCs evidently suggests the derivation or origination of BTSCs from NSCs. It is thus particularly interesting to study the biological behaviors of BTSCs through comparison with NSCs.

Yuan et al.[13] reported that tumor sphere could re-form after induction of differentiation if re-subjected to defined stem medium. In this study, we found BTSCs could re-form tumor spheres even in the persistent differentiation condition. The expression of differentiated and undifferentiated markers seemed to coincide with the morphological changes of BTSCs in the differentiation assay. To sum up, the differentiation process of BTSCs seemed unstable (floating down and up). Actually, both the morphological changes and the shifting of cell marker expression denoted BTSCs' 

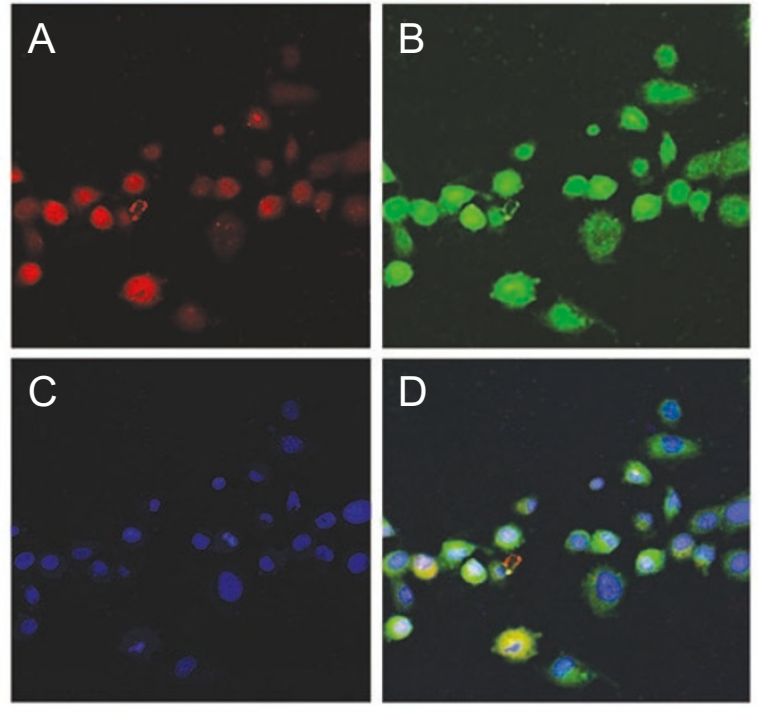

1
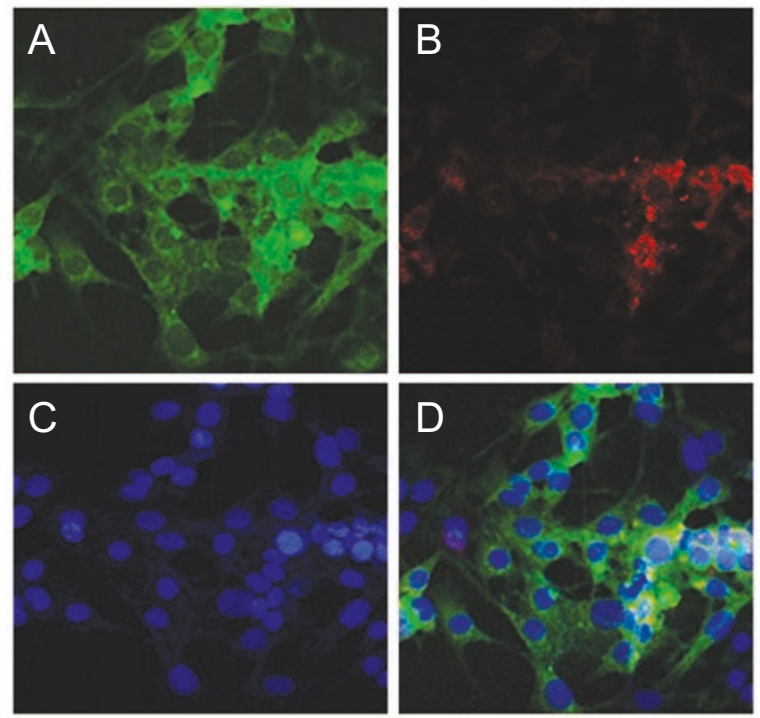

3

intrinsic capacity to maintain undifferentiated state or resist differentiation. Both positive and negative regulators, including internal and external signals, control the cell fate of stem cells. Negative regulators keep the stem cells undifferentiated, while positive regulators instruct the commitment and differentiation of stem cells [27-28]. In the same differentiation condition, NSCs differentiated to the mature neural cell types, while BTSCs rebounded to keep undifferentiated status. This difference indicates internal signaling pathway regulating differentiation has been severely shifted by accumulated gene mutation(s) in BTSCs; in other words, genes giving rise to differentiation have been suppressed by genes keeping stem cell undifferentiated. To determine the genes responsible for the dedifferentiation through arrayed comparative genome hybridization and other methods is of

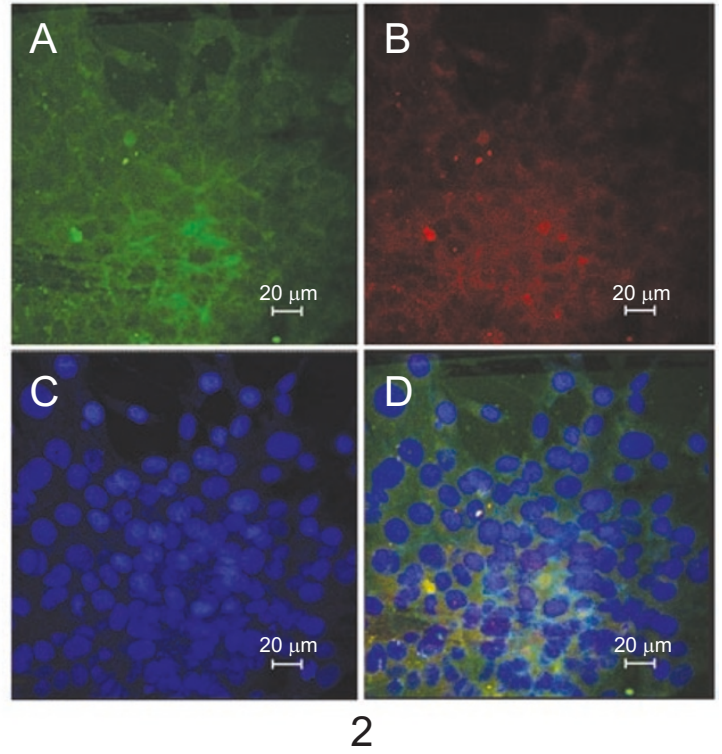

Figure 5 Immunofluorescence performed on BTSCs during differentiation was checked with laser scanning confocal microscope (magnification $800 \times$ ), with nuclei of BTSCs stained by DAPI (in blue, shown in the C picture of all the three groups of photos". (1) At the beginning of differentiation, cells were stained for undifferentiated marker CD133 (in red, A) and nestin (in green, B). (D) costaining of CD133 and nestin when pictures were merged. (2) Nestin (in green, A) and CD133 (in red, B) staining was still positive after differentiation for 10 days. (D) Costaining of nestin and CD133was showed, but nestin staining was predominant. (3) Ten days after differentiation, nestin staining (in green), GFAP staining (in red) and costaining of nestin and GFAP were demonstrated in (A), (B) and (D) respectively.

great significance in probing the molecular mechanism of brain tumor initiation. Abnormal differentiation is characteristic of and responsible for the malignance of glioma. The strategy aimed to induce glioma cells into terminal differentiation has been carried out in our center in the past years; however, only temporary and incomplete differentiation was achieved [29-31]. The differentiation characters that BTSCs display make it reasonable to conjecture that BTSCs are responsible for the unsatisfactory outcome of differentiation-inducing treatment. How to induce BTSCs to mature status is critical in this therapy modality.

The identification of BTSC is due to, at least in part, the forming of tumor sphere by BTSCs. However, BTSCs were also identified from adherent and monolayer tumor cell population [6]. Then, what makes the tumor sphere? 

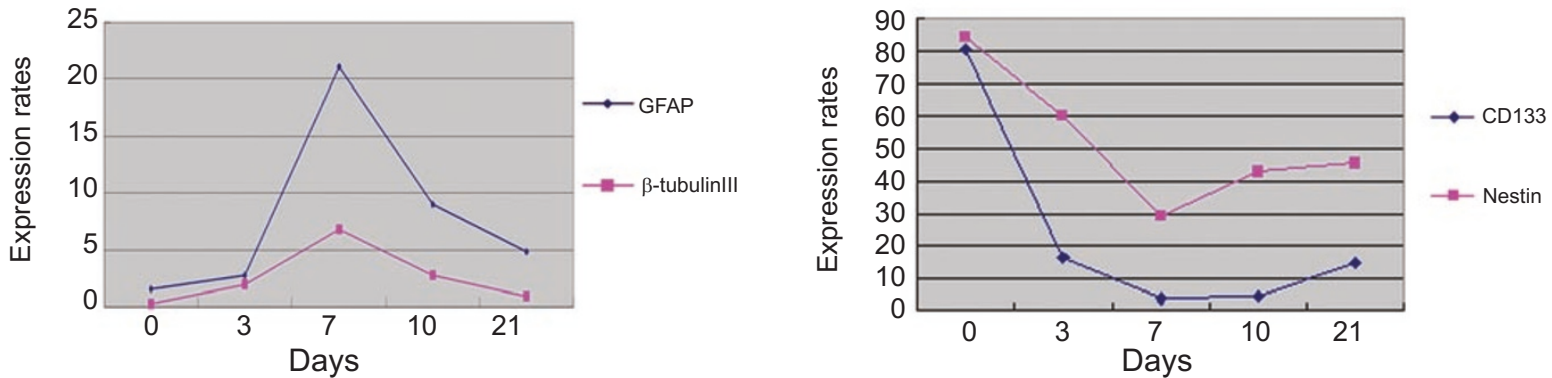

Figure 6 In differentiation condition, the expression rates of differentiated cell markers GFAP and $\beta$-tubulinIII in BTSCs were found to keep rising for the first week, but declined thereafter (in the left). During the corresponding period, the expressing tendency of undifferentiated cell markers CD133 and nestin was perfectly inverse to that of differentiated cell markers (in the right). The expression rate refers to the number of marker positive cells divided by total number of examined cells.

Table 1 Expression rates of differentiated cell surface markers

\begin{tabular}{cccccc}
\hline & Time $(\mathrm{d})$ & CD133 & Nestin & GFAP & $\beta$-TubullinIII \\
\hline \multirow{2}{*}{ BTSC } & 0 & $80.76 \pm 1.22$ & $84.12 \pm 1.83$ & $1.55 \pm 0.13$ & $0.26 \pm 0.11$ \\
& 3 & $16.33 \pm 0.89$ & $60.25 \pm 1.08$ & $2.79 \pm 0.25$ & $1.96 \pm 0.22$ \\
& 7 & $3.65 \pm 0.17$ & $28.99 \pm 1.26$ & $21.12 \pm 0.61$ & $6.74 \pm 0.54$ \\
& 10 & $4.59 \pm 0.33$ & $42.73 \pm 1.95$ & $4.98 \pm 0.87$ & $2.79 \pm 0.19$ \\
& 21 & $14.63 \pm 1.16$ & $45.46 \pm 1.27$ & $1.77 \pm 0.34$ & $0.19 \pm 0.11$ \\
NSC & 0 & $78.79 \pm 1.95$ & $86.36 \pm 1.67$ & $88.94 \pm 1.23$ & $11.94 \pm 0.36$ \\
\hline
\end{tabular}

In BTSCs' differentiation assay, growth into adherent status was accompanied by the decrease of CD133 and nestin, while reforming of tumor sphere concurred with the increase of CD133 and nestin. We also separated BTSCs from tumor spheres and monolayer tumor cell population, and found that the frequency of BTSCs in tumor sphere was almost twice as much as that in monolayer cell population ( $4 \%$ versus $2 \%$ ). Taken together, the proportion of BTSCs in tumor cell population may account for the formation of tumor sphere; however, more studies are necessary to get a clear answer.

\section{Acknowledgments}

The current research was supported by National Natural Science Foundation of China (No. 30371457 and 30400457). We would express our thanks to the staff of Nerve Regeneration Key Laboratory of Jiangsu Province, Nantong University, who kindly helped to perform the Immunofluorescent analysis with laser scanning confocal microscope.

\section{References}

1 Reya T, Morrison SJ, Clarke MF, Weissman IL. Stem cells, cancer, and cancer stem cells. Nature 2001; 414:105-111.

2 Dick JE. Breast cancer stem cells revealed. Proc Natl Acad Sci USA 2003; 100:3547-3549.

3 Wulf GG, Wang RY, Kuehnle I, et al. A leukemic stem cell with intrinsic drug efflux capacity in acute myeloid leukemia. Blood 2001; 98:1166-1173.

4 Singh SK, Clarke ID, Terasaki M, et al. Identification of a cancer stem cell in human brain tumors. Cancer Res 2003; 63:58215828.

5 Galli R, Binda E, Orfaneli U, et al. Isolation and characterization of tumorigenic, stem-like neural precursors from human glioblastoma. Cancer Res 2004; 64:7011-7021.

6 Kondo T, Setoguchi T, Taga T. Persistence of a small subpopulation of cancer stem-like cells in the $\mathrm{C} 6$ glioma cell line. Proc Natl Acad Sci USA 2004; 101:781-786.

7 Ignatova TN, Kukekov VG, Laywell ED, et al. Human cortical glial tumors contain neural stem-like cells expressing astroglial and neuronal markers in vitro. Glia 2002; 39:193-206.

8 Hemmati HD, Nakano I, Lazareff JA, et al. Cancerous stem cells can arise from pediatric brain tumors. Proc Natl Acad Sci USA 2003; 100:15178-15183.

9 Singh SK, Hawkins C, Clarke ID, et al. Identification of human brain tumour initiating cells. Nature 2004; 432:396-401.

10 Galdersisi U, Cipollaro M, Giordano A. Stem cells and brain cancer. Cell Death Differ 2006; 13:5-11.

11 Sanai N, Alvarez-Buylla A, Berger MS. Neural stem cells and the origin of gliomas. N Engl J Med 2005; 353:811-822. 
12 Clevers H. Stem cells, asymmetric division and cancer. Nat Genet 2005; 37:1027-1028.

13 Yuan X, Curtin J, Xiong Y, et al. Isolation of cancer stem cells from adult glioblastoma multiforme. Oncogene 2004; 23:93929400.

14 Zhang QB, Huang Q, Lan Q. Research progress in brain tumor stem cell. Natl Med J China 2005; 85:1868-1870.

15 Fomchenko EI, Holland EC. Stem cell and brain cancer. Exp Cell Res 2005; 306:323-329.

16 Nakano I, Kornblum HI. Brain tumor stem cells. Pediatr Res 2006; 59:54R-58R.

17 Gilbertson RJ. Brain tumors provide new clues to the source of cancer stem cells: does oncology recapitulate ontogeny? Cell cycle 2006; 5:135-137.

18 Shemer G, Podbilewicz B. The story of cell fusion: big lessons from little worms. Bioessays 2003; 25:672-682.

19 O'Malley K, Scott E W. Stem cell fusion confusion. Exp Hematol 2004; 37:131-134.

20 Ogle BM, Cascalho M, Platt JL. Biological implications of cell fusion. Nat Rev Mol Cell Biol 2005; 6:567-575.

21 Brustle O, McKay RD. The neuroepithelial stem cell concept: implications for neuro-oncology. J Neurooncol 1995; 24:57-59.

22 Holland EC. Progenitor cells and glioma formation. Curr Opin Neurol 2001; 14:683-688.
23 Stecca B, Ruiz i Altaba A. Brain as a paradigm of organ growth: Hedgehog-Gli signaling in neural stem cells and brain tumors. J Neurobiol 2005; 64:476-490.

24 Polakis P. Wnt signaling and cancer. Genes Dev 2000; 14:18371851.

25 Baron M. An overview of the Notch signaling pathway. Semin Cell Dev Biol 2003; 14:113-119.

26 Beachy PA, Karhadkar SS, Berman DM. Tissue repair and stem cell renewal in carcinogenesis. Nature 2004; 432:324-331.

27 Jessell TM. Neuronal specification in the spinal cord: inductive signals and transcriptional codes. Nat Rev Genet 2000; 1:2029.

28 Kennea NL, Mehmet H. Neural stem cells. J Pathol 2002; 197:536550.

29 Li XN, Du ZW, Huang Q. Modulation effects of hexamethylene bisacetamide on growth and differentiation of cultured human malignant glioma cells. J Neurosurg 1996; 84:831-838.

30 Li XN, Du ZW, Huang Q, Wu JQ. Growth-inhibitory and differentiation-inducing activity of dimethylformamide in cultured human malignant glioma cells. Neurosurgery 1997; 40:1250-1259.

31 Huang Q, Sun LJ, Dong J, et al. Preliminary study on differentiation-inducing associated genes of human brain glioma cells in vitro. Ai zheng 2003; 22:673-679. 